\title{
Corrosion inhibition by self assembling nano films
}

\author{
Susai Rajendran \\ RVS School of Engineering and Technology, Dindigul-624005, India \\ Email: susairajendran@gmail.com
}

Many researches are going on in the field on nanotechnology, because, nanoparticles have special properties (optical, electrical etc.,) and special applications in many fields. The synthesis and characterization of nanoparticles of silver and gold have been widely studied. Self assembling nano films have ordered structure. Polymeric nanoparticles have also been studied. Silver nanoparticles can be arranged in wire forms also. It has been observed that self assembled nano films offer better corrosion inhibition efficiency. Inhibition of corrosion of mild steel has been widely studied. Alkane thiols have been used to prevent corrosion of mild steel by formation of monolayers. Alkane mono phosphonic acid films have been used to prevent the corrosion of iron. Further, phosphonic acids, carboxylic acids, hydroxamicacid, and sulphonic acids can form self assembling mono layers. Such mono layers have been formed on stainless steel, aluminium, titanium and copper oxide surfaces. The present work is under taken to form self assembling mono layers of molecules such glycine[1], SDS, some natural products and inorganic compounds on mild steel surface and also aluminium surface and investigate the nature of monolayers by FTIR spectroscopy and AFM study. Contact angle measurements have also been done. The corrosion inhibition studies have been carried out by weight loss method, polarization study, AC impedance spectra and Fluorescence spectra. The concept of nano films will be explained in terms of lotus effect also.
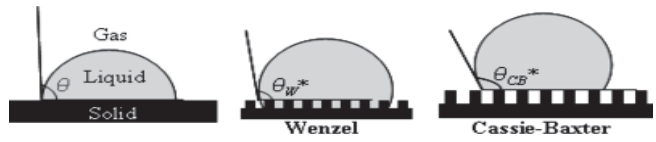

Figure 1: Contact angle in various models
A liquid droplet rests on a solid surface and is surrounded by gas (Figure 1). The contact angle, $\theta_{\mathrm{C}}$, is the angle formed by a liquid at the three-phase boundary where the liquid, gas, and solid intersect. Correlation between hydrophobicity and corrosion inhibition efficiency will be explained (Figure 2).

The relation between lotus effect (Figure 3), self cleaning effect and corrosion inhibition will be explained.

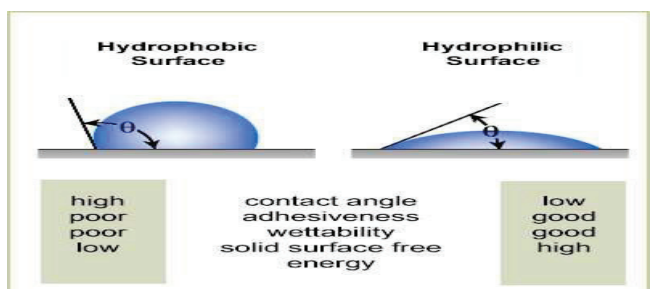

Figure 2: Hydrophobic and hydrophilic surfaces

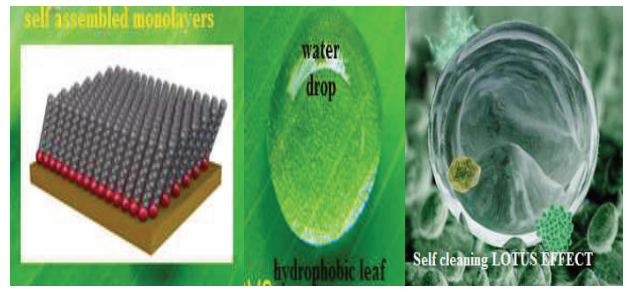

Figure 3: Lotus effect and self cleaning effect

High contact angle, high adhesiveness, poor wettability and low surface free energy increase hydrophobicity of a surface which enhances the corrosion resistance of a film formed on a metal surface.

\section{Reference}

1. Corrosion Inhibition by Self Assembling Monolayers on Carbon Steel, S. Gowri, J Sathiyabama, Susai Rajendran*, R M. Joany, J Jeyasundari and TUmasankareswari, Innovations in Corrosion and Materials Science, 2015, 5, 113-117 